\title{
The SER-CAT Virtual Beamline: Providing light when YOU need it in your home lab
}

\author{
Z Jin ${ }^{1}$, J Gonczy ${ }^{1}$, J Fait ${ }^{2}$, Z Fu $^{1}$, J Chrzas ${ }^{1}$, J Rose ${ }^{3}$, B Wang ${ }^{4}$ \\ ${ }^{1}$ SER-CAT/University of Georgia, Argonne, IL, ${ }^{2}$ SER-CAT BMB, UGA, ${ }^{3}$ SER-CAT/University of \\ Georgia, Athens, GA, ${ }^{4}$ University of Georgia \\ zmjin@anl.gov
}

The SER-CAT Virtual Beamline: Providing light when YOU need it in your home lab Zhongmin Jin, John Gonczy, James Fait, Zheng-Qing "Albert" Fu, John Chrzas, John P. Rose and B.C. Wang. Southeast Regional Collaborative Access Team, Advanced Photon Source and the Department of Biochemistry and Molecular Biology University of Georgia, Athens, GA 30602. Since 1999, SER-CAT has been working towards the concept of providing its members with a "Virtual Beamline, which could be integrated into their daily workflow much like your X-ray lab down the hall. SER-CAT beamlines 22ID and 22BM were designed and built for remote operation, we began investigating robotic crystal mounting automation in 2000 with Oceaneering Space Systems. In 2003, a highly modified Berkeley ALS Automounter was installed on 22BM. Using 22BM as a user-based testbed, the beamline and experiment control interface SERGUI was continually modified until a reliable, robust and user-friendly system was achieved. In 2006, the SER-CAT Virtual Beamline came online providing remote crystal screening and data collection capability on both 22ID (capacity 430 crystals) and 22BM (capacity 96 crystals). The Virtual Beamline includes software integration as well. The SERGUI beam line control interface allows the remote user full control of the beamline from their home lab including beamline/goniometer optimization, wavelength selection, fluorescence scans, automatic crystal centering and rastering, automated crystal screening and MAD/SAD/Helical data collection. Today over $95 \%$ of SER-CAT members routinely collect data remotely. To assist remote users in the efficient use of their beamtime, SER-CAT has implemented 12-hour shifts with 16-hours/day of on-site user support. Multiple Access Time (MAT) shifts have also been made available to SER-CAT members for fast turnaround and flexible data collection capabilities. SER-CAT also provides its members access to on-the-fly automated data processing using XDS, KYLIN and DIALS. HKL2000, XDS, MOSFLM, and DIALS are also available for manual data processing. All user data can be remotely, quickly, reliably and securely downloaded from SER-CAT's Globus/GridFTP archive server to the users' home systems anywhere and anytime. An overview of SER-CAT's Remote Access Program including robotics, beamline/experiment control, automated data processing and structure determination will be described and discussed. Work supported by the SER-CAT Member Institutions, University of Georgia Research Foundation, The National Institutes of Health (S10_RR25528 and S10_RR028976) and the Georgia Research Alliance.

Acta Cryst. (2020). A76, a170 